\title{
LES RECHERCHES EN ÉDUCATION AU QUÉBEC: ENTRE HISTOIRE ET SOCIOLOGIE ${ }^{1}$
}

\author{
Thérèse Hamel ${ }^{2}$ \\ Féconder le passé en créant l'avenir, \\ que tel soit mon préscnt. \\ Nietzsche
}

RÉSUMÉ : Au moment où l'on fait appel de plus en plus à l'interdisciplinarité, les rapports entre Ia Sociologie et l'Histoire sont à une étape charnière de leur développement. Autrefois relativement indépendantes les unes des autres, ces deux disciplines tentent de s'influencer, de dépasser leur barrière respective, tout en gardant leur spécificité, pour en arriver à une rneilleure compréhension des phénomènes sociaux. Dans un contexte où les Sciences Humaines cherchent à se redéfinir une place dans le champ social, il importe de réfléchir à fond sur l'apport heuristique de deux secteurs majeurs au sein des Sciences Humaines, l'Histoire et Ia Sociologie.

Mots Clés: Histoire; Sociologie; Interconections

\section{RESEARCH ON EDUCATION IN QUEBEC: BETWEEN HISTORY AND SOCIOLOGY}

\begin{abstract}
At the moment, when attention is called to the question of interdisciplinary, it can be observed that the relationships between Sociology and History have a particular evolution. They are independent disciplines and they are trying to influence one each other, bypassing their limits, although maintaining their specificity, with the objective of a better understanding of social phenomenon. Human Sciences are redefining their place in social area and it is important to think about the heuristic contribution of these two major sectors : History and Sociology.
\end{abstract}

Key Words : History; Sociology; Interconnections

On connaît les appels répétés de Braudel (1969) pour un marché commun des sciences de l'homme et la brisure des clivages entre disciplines Je suis particulierement sensible à une telle approche . À mon avis, Histoire et Sociologie représentent deux outils fondamentaux de compréhension de l'institution complexe qu'est l'école. Cet article se penchera plus spécifiquement sur l'évolution des rapports entre Histoire et Sociologie dans le secteur de l'éducation au Québec. Partant de la production scientifique en Histoire et en Sociologie de l'Éducation au Québec, je tenterai de dégager l'apport

\footnotetext{
${ }^{1}$ Artigo recebido para publicação em novembro de 1999; aceito em junho de 2000.

${ }^{1}$ Endereço para correspondência: Départment des Fondements et Pratiques em Éducation- Faculte des Sciences de l'Éducation- Université de Laval - Quebec - G1K7P4 - Canadá.

E-mail Therese.Hamel@fse.ulaval.ca
}

Paidéia, FFCLRP-USP, Rib. Preto, jan/julho/2000. respectif de chacun de ces deux champs du savoir, leur spécificité pour enfin présenter des pistes de réflexion pour an arrimage plus fin entre Histoire et Sociologie. Ce texte ne prétend nullement être un bilan exhaustif du travail qui s'est fait au cours de ces dernières années ${ }^{1}$. Il se veut plutôt une esquisse réflexive sur les rapports qu'entretiennent Histoire et Sociologie dans le domaine éducatif au Québec. Je me baserai principalement sur quelques bilans historiographiques et sociologiques déjà établis ainsi que sur des travaux qui m'apparaissent représentatifs de certains courants majeurs dans ces deux champs disciplinaires.

\footnotetext{
'Em effet, um premier repérage nous a vite convaincu de l'impossibilité de réaliser ce travail dans lê temps imparti, étant donné l'importance et la quantité des travaux faits dans ces deux domaines. Lê nombre d'écrits touchant l'Histoire de l'Éducatin à lui seul est impressionnant.
} 


\section{Les rapports entre Histoire et Sociologie au Québec}

En 1990, lors d'une conférence au congrès de l'Association Canadienne d'Histoire de l'Éducation, Micheline Dumont exposait avec acuité et lucidité certaines tendances caractérisant les travaux en Histoire de l'Éducation. Elle estime d'une part que les premiers travaux en Histoire de l'Éducation au Québec, publiés en général par les élites de l'époque, se sont longtemps acharnés à vouloir montrer que nous possédions le meilleur système scolaire du monde.

On n'y trouve principalement que des panégyriques, des plaidoyers pro damo et des récits souvent apologétiques sur la clairvoyance et le dévouement des initiateurs. Dans ces ouvrages également, il existe une myopie flagrante face aux lacunes d'un système d'enseignement qui n'avait ni voulu ni réussi à se démocratiser (Dumont, 1990, p. 211).

A contrario, à partir de la Révolution tranquille, on a tenté de nous prouver que nous avions le plus mauvais système d'éducation. Selon cette historienne, nous avons été portés par ces deux "interprétations manichéennes qui ont bien des chances de n'être vraies ni l'une ni l'autre. Au demeurant, elles ne sont scientifiques ni l'une ni l'autre» (Dumont, 1990, p. 211). Afin d'expliquer ce sous-développement des travaux en Histoire de l'Éducation au Québec, elle invoque d'abord une cause structurelle, soit le peu de place accordée à l'Histoire de l'Éducation dans les Facultés d'Éducation. L'autre facteur serait la polarisation apportée par la Révolution tranquille sur notre façon d'entrevoir l'évolution de notre système éducatif, polarisation qui, toujours selon cette auteure, ferait en sorte qu'après ce sursaut important de notre histoire scolaire, on ne se serait désormais plus penché sur ce qui se faisait avant 1960 .

Cette hantise des origines oblitère souvent la compréhension globale des phénomènes importants qui se sont produits avant 1960 et qui ont constitué des ruptures significatives, en dépit de structures apparemment inchangées du système scolaire entre 1841 et 1943 (p. 214)

Selon cette historienne, le "syndrome Révolution tranquille» a provoqué des erreurs de perspectives importantes. Elle critique fort justement les cadres d'analyse trop simplistes, par exemple l'opposition dichotomique Église-État, ou encore une vision passéiste et archaïque du temps révolu. Or il est vrai que l'on ne peut se contenter de juger le passé à la seule lumiére de cé point d'arrivée qu'est la Révolution tranquille. Devant cette amnésie historique, le domaine de la Sociologie de l'Éducation a donc investi le champ des études en éducation, voire même celui des études historiques. "C'est donc la Sociologie qui s'est constituée le maître d'oeuvre théorique de la recherche en éducation» (p. 214). On se retrouve ainsi devant le paradoxe actuel: «pléthore d'études sur la période très récente et unanimité factice sur l'interprétation à donner à la période précédente» (p. 215).

Bien qu'étant en partie d'accord avec les éléments généraux de cette analyse je constate qu'au Québec, nous sommes en train d'évoluer très rapidement vers une redéfinition des champs respectifs de l'Histoire et de la Sociologie de l'Éducation. À partir d'un survol des travaux faits en Histoire de l'Éducation au Québec, j'examinerai d'abord le penchant vers une approche sociologique ${ }^{2}$.

\section{De l'Histoire à la Sociologie de l'Éducation}

Les critiques fort pertinentes exprimées par Micheline Dumont n'invalident pas, loin s'en faut, l'ensemble des travaux faits dans une perspective historique en éducation. S'il est vrai que les intervenant(e)s de l'époque ont énormément publié pour defendre leurs points de vue, nous disposons de travaux importants qui jettent un regard fort éclairant sur notre histoire scolaire. Parmi les travaux qui ont marqué l'Histoire de l'Éducation, on ne peut passer sous silence ceux de Louis-Philippe Audet (1951, 1964, 1971), qui se voulaient de grandes synthèses montrant l'évolution de notre histoire scolaire.

\footnotetext{
2 Aontoine Prost dans son analyse des rapports entre Histoire et Sociologie en éducation em France insite beaucoup plus sur l'approche privilégiée par les chercheurs-es que sur leur secteur disciplinaire ou leur appartenance à um groupe donné, que ce soit par leur formation ou encore leur lieu de travail.
}

Paidéia, FFCLRP-USP, Rib. Preto, jan/julho/2000. 
D'ailleurs son ouvrage Histoire de l'Enseignement au Québec représente toujours à ce jour la seule synthèse existante en Histoire de l'Éducation (1971). Depuis la production de cette synthèse, plusieurs ouvrages explorent à leur façon les relations ÉgliseÉtat. Notre compréhension de certains enjeux scolaires est enrichie par des travaux comme ceux de Nadia Fahmy-Eid (1978) sur les ultramontains, l'ouvrage de Bemard Lefebvre (1980) intitulé L'école sous la mitre ou encore la thèse de Ruby Heap (1986) qui contribue aussi fortement à éclairer notre connaissance des relations Église-État pendant la période qui s'échelonne de la fin du siècle dernier à 1920.

En outre, des filières spécifiques de notre appareil scolaire ont été scrutées à la loupe des historiens. Les travaux de Claude Galarneau (1978) sur les collèges classiques, de Jean-Pierre Charland (1982) sur l'enseignement professionnel et technique, de Nicole Thivierge (1982) sur les écoles ménagères, des thèses comme celle de Marîse Thivierge (1981) sur les institutrices laïques, ou encore de Roland Piquette (1973) sur les programmes de formation des maîtres, ajoutent à l'édifice des réalisations en Histoire de l'Éducation. Quant à Nadia Fahmy-Eid et Micheline Dumont, elles ont ouvert l'énorme chantier qu'est l'Histoire de l'Éducation des filles. Enfin, il ne faudrait pas oublier la production scientifique chez les anglophones. Signalons ici, à titre d'exemples, les travaux de Roger Magnuson (1969), de Nathan Mair (1980; 1981), ainsi que ceux de Norman Henchy et Donald Burgess (1987).

Les travaux forts importants d'Histoire de l'Éducation mentionnés précédemment levaient le voile sur des facettes cachées de notre appareil scolaire. Mais, selon Micheline Dumont (1990, p. 216): «Force est de constater chez nous l'absence de débats théoriques éclairants, spécifiques à la réalité québécoise en Histoire de l'Education [par rapport aux collègues anglo-canadiens].»

Il est vrai que nous n'avons pas directement été confrontés aux débats apportés entre autres par le courant «révisionniste ${ }^{3}$ » du Canada anglais qui cherchait à réinterpréter le rôle de l'État en éducation. Ce courant, important et soutemu par la vivacité de toute l'équipe de l'Ontario Institute for the Studies in Education (OISE) à Toronto, regroupée autour de

${ }^{3}$ Ls signification du terme 'révisionniste' ná pas ici lê sens quón lui donne em France em faisant référence aux partis politiques de la gauche.

Paidéia, FFCLRP-USP, Rib. Preto, jan/julho/2000.
Michael Katz, s'opposait à des interprétations historiques antérieures, qualifiées de progressivistes, qui voyaient dans l'Histoire de l'Éducation une lente mais constante progression vers l'amélioration de notre système d'enseignement (Phillips, 1957). A contrario, l'approche de Michael Katz (1971) considère plutôt le système d'éducation comme fondamentalement sexiste, bureaucratique et dispensant un enseignement de classe, particulièrement dans son ouvrage Class, Bureaucracy and School. The illusion of Educational Change in America.

Sans entrer ici dans les débats théoriques et historiographiques qui ont eu cours à ce sujet, ce qui nous amènerait trop loin, contentons-nous de signaler qu'au Québec, nos interprétations historiques ne s'imbriquaient pas dans des débats théoriques de ce genre (Wilson, 1984). Selon Micheline Dumont, les historiens et historiennes ont peu contribué au niveau théorique. Les sociologues de l'éducation auraient occupé cet espace laissé vacant, prenant en quelque sorte le devant de la scène. C'est en définitive ces derniers-ères qui seraient le plus avancés théoriquement et conceptuellement. Toujours selon cette auteure, la contribution de la Sociologie de l'Éducation s'est fait sentir au niveau théorique, plus particulièrement au niveau du cadre conceptuel et du cadre d'analyse, les sociologues ayant en outre investi le champ de l'Histoire de l'Éducation, pendant la période qui suit immédiatement la Révolution tranquille. Examinons maintenant les rapports entre Histoire et Sociologie de l'Éducation au Québec, en optant pour la démarche inverse. Nous partirons donc des travaux de la Sociologie de l'Éducation pour présenter quelques cas de sociologues qui ont travaillé avec une approche historique.

\section{De la Sociologie de l'Éducation vers l'Histoire}

S'il y eut des appels d'historiens à un recours aux Sciences Humaines, à l'instar de Braudel nous conviant à un marché commun des sciences de l'homme, il y eut aussi de la part des sociologues de l'éducation, et plus particulièrement d'un courant appelé la «Nouvelle Sociologie de l'Éducation» (NSE), un appel "inverse visant à donner à la Sociologie un éclairage, voire un ancrage historique. Dans son livre intitulé Social Analysis of Education 
after the New Sociology of Education, Wexler (1987) cherche à situer le courant de la nouvelle Sociologie de l'Éducation dans son contexte historique et sociétal. Alors que la Sociologie antérieure faisait miroiter les mirages de la «méritocratie» et la possibilité de s'élever dans l'échelle sociale grâce à l'éducation, insistant sur les phénomènes de mobilité, la Nouvelle Sociologie de l'Éducation (NSE) s'est concentrée sur la théorie de la reproduction sociale des inégalités. Cet auteur critique la non prise en compte par ce courant théorique, des transformations sociales opérées par l'école. Malgré les modifications apportées par la NSE, Wexler tente de montrer qu'elle reste néanmoins ancrée dans ses limites, entre autres dans sa capacité à interpréter le changement social. Même lorsqu'elle fait intervenir le conflit, cette notion est réduite à la notion de résistance, ce qui, au dire de Wexler, le ramène à la façon fonctionnaliste d'entrevoir le changement social. On se retrouve donc inévitablement dans le cadre général de la reproduction, même lorsque les auteurs voulaient $s$ 'en départager.

Pour Wexler, les limites de la NSE résident principalement dans la dichotomie qu'elle a souvent utilisée entre l'ancien et le nouveau, une catégorisation binaire basée sur une négation de nature «anhistorique». Pour Michael Apple; un des fondateurs de la NSE, il ne faut pas oublier que la société «is the product of historical collective action». Selon lui, «We must first reconnect theory, research and practice to hisiorical movements in society and education» (Wexler,1987,p.xiii). Or la NSE analysait, par exemple, les contenus scolaires, le curriculum; en oubliant que ces notions étaient historiquement constituées. Pour cet auteur, la nonintégration de la perspective historique dans les recherches en éducation et particulièrement dans la NSE, donne l'illusion que les choses sont immuables, qu'elles sont prises pour argent comptant. Le changement social y apparaît donc comme plus difficile ${ }^{4}$. Malgré elle, la nouvelle Sociologie de l'Éducation serait elle-même devenue idéologie en adoptant des théories anhistoriques: "When they spoke of educational practice, it was in a language that ignored trie historical piactice of the social movement that they were intellectually recouping and revising "(Wexler,

\footnotetext{
${ }^{4}$ Une autre critique selon lui vient du fait que la NSE na pas réussi a comprendre le role de l'éducation dans la transformation sociale et na pas réussi à contrecarrer ce quíl apppelle le courant de la nouvelle droite.
}

1987, p. 78).

En vue de dépasser les limites identifiées, Wexler propose de replacer la Sociologie de l'Éducation comme étant une activité culturelle, ancrée dans un milieu social donné. En tant que discours et pratique produite par un groupe social donné, elle doit être yue comme une action historique, elle-même produit de son histoire et intégrée dans une pratique de changement social inscrite historiquement. Il ira jusqu'à faire une étude de l'évolution propre de la discipline ou du champ de la Sociologie de l'Éducation pour en montrer les limites internes. C'est en quelque sorte à une socio-histoire de la NSE que Wexler nous convie.

Nous sommes donc devant deux appels à l'intégration des disciplines. D'une part et depuis longtemps, des historiens comme Braudel insistent sur l'importance de la longue durée, celle-ci permettant de relativiser notre regard sur le présent. D'autre part, la Sociologie de l'Éducation et certains représentants de la nouvelle Sociologie de l'Éducation, à l'instar de Philip Wexler, cherchent à dépasser les limites de leur discipline trop ancrée dans le présent, en intégrant là aussi, d'une certaine façon, la longue durée. Est-ce pure coïncidence si Nouvelle Histoire et Nouvelle Sociologie de l'Éducation se rejoignent en s' interpellant mutuellement? La longue durée semblé constituer le fil directeur aux deux disciplines.

\section{La réponse aux appels à l'interdisciplinarité: quelques réalisations}

Les appels à l'interdisciplinarité dont nous avons parlé ont touché le monde de l'éducation. Viviane Isambert-Jamati en France et André Petitat au Québec en représentent d'excellents exemples. Qu'en est-il de la Sociologie de l'Éducation au Québec? À l'instar d'autres formations sociales, nous avons été influencés par les pirincipaux courants ou paradigmes sociologiques qui ont fasciné les sociologues du inonde entier.

\section{La Sociologie de l'Éducation au Québec}

L'évolution de la Sociologie de l'Éducation au Québec doit être replacée par rapport aux grands courants qui ont influencé cette discipline. Elle doit aussi être située dans le contexte particulier de l'évolution du Québec. Dans un court texte sur les

Paidéia, FFCLRP-USP, Rib. Preto, jan/julho/2000. 
recherches effectuées en Sociologie de l'Éducation au Québec, Pierre Dandurand (1984) montre clairement comment la remisé en question des analyses fonctionnalistes et structuro-fonctionnalistes doit être mise en relation avec l'amère constatation de l'érosion des acquis de la Révolution tranquille (Dandurand,1984, p.69). On assiste alors à la production d'études et analyses très critiques des réalisations de notre appareil scolaire et particulièrement de son rôle dans la reproduction des classes sociales. L'ouvrage de Claude Escande intitulé Les classes sociales au Cégep, ou encore les manifestes et études de la Centrale des enseignants du Québec (CEQ) aux titres aussi évocateurs que L'école au service de la classe dominante, illustrent de façon éloquente le ton des approches utilisées.

Dans son article, Pierre Dandurand (1984) signale alors que les trois quarts des recherches en Sociologie de l'Éducation se situent dans deux champs de recherches principaux, soit l'accès différentiel des groupes sociaux à l'enseignement (avec un élargissement des perspectives) et les relations éducation-emploi. Six ans plus tard, dans un texte beaucoup plus volumineux écrit en 1990 avec Roland Ouellet, il publie un bilan bibliographique de la recherche en Sociologie de l'Éducation produite entre 1976 et 1986 . On y remarque l'élargissement des perspectives et la prise en compte de nouveaux champs de recherches intégrant dorénavant entre autres les effets liés au sexe, à l'ethnie et à l'âge, de même que l'émergence de nouvelles pratiques et l'utilisation de nouvelles méthodologies. Les principaux thèmes abordés sont: 1) L'accessibilité à l'enseignement; 2) L'ěducation et l'emploi; 3) Les politiques scolaires; 4) L'impact de l'organisation scolaire sur la socialisation et, enfin, 5) L'apprentissage et la profession enseignante (Dandurand et Ouellet, 1990).

Les trois derniers ternies, en émergence quelques années auparavant, prendront leur essor à l'aube des années quatrevingt-dix. On assiste aussi, selon ces auteurs, à l'éclatement des objets d'analyse, ainsi qu'à un requestionnement théorique et méthodologique. À l'approche par grandes enquêtes qui caractérisait les années soixante-dix et le début des années quatre-vingt, s'ajoutent des recherches de nature plus qualitative. Une association provinciale (l'Association pour la recherche qualitative) regroupe

Paidéia, FFCLRP-USP, Rib. Preto, jan/julho/2000. d'ailleurs les adeptes de ces méthodes et la présence des chercheurs-es en éducation y est fort importante. La Sociologie de l'Éducation dans les quarante dernières années serait passée du paradigme fonctionnaliste, à un paradigme plus radical allant au marxisme, puis à un désenchantement des possibilités du système scolaire vers une attention particulière sur ce que Dandurand et Ollivier (1987) appellent la «boîte noire de l'école».

Nous avons signalé précédemment l'amnésie historique des recherches en Sociologie de l'Éducation, du moins au cours des années soixante$\mathrm{dix}^{5}$. Dans le chapitre intitulé "Notes sur le développement de champs limitrophes à la sociologie de l'éducation», Dandurand et Ouellet (1990) disaient: "Le mouvement de la recherche en sociologie de l'éducation se poursuit simultanément, bien que trop souvent en parallèle, au développement de la recherche dans des domaines limitrophes comme l'histoire, l'économie, les sciences politiques» (p. 67). Pourtant, les sociologues ne se désintéressent pas nécessairement de l'histoire, quoique ce mouvement puisse apparaître comme récent. À l'instar de Viviane Isambert Jamati et d'André Petitat, certains sociologues ont eu un penchant pour l'histoire.

Que se passe-t-il quand des sociologues font de l'histoire?

Des recherches fort intéressantes failes par des sociologues de l'éducation ont recours à une approche historique. Je prendrai ici l'exemple des travaux de Paul-André Turcotte $(1981 ; 1985 ; 1988)$ sur les Frères enseignants. Grâce aux recherches fouillées de cet auteur qui utilise simultanément des concepts sociologiques et la méthode historique pour appuyer et étayer sa démonstration, nous pouvons maintenant avancer dans la réinterprétation de lá Révolution tranquille et ainsi éviter les écueils signalés par Micheline Dumont. La dynamique des rapports Église-État prend alors un tout autre relief. Les tentatives des Fières éducateurs pour

\footnotetext{
${ }^{3}$ Même au début des années quatre-vingt, au moment où je démarrais um projet de recherche sur l'évolution de la formation des enseignants et enseignabtes au Québec, plusieurs questionnaient ou du moins semblaient douter de la pertinence d'une approche qui intègre um retour sur l'histoire des enseignants et enseignantes. Est-il besoin de dire que ce n'est plus lê cãs aujourd'hui. La necessite.
} 
démocratiser l'enseignement, mises en relief par P.: A. Turcotte, ajoutent quelques bémols aux idées triomphalistes des artisans des réformes des années soixante et de chercheurs-es qui avaient tendance à voir en blanc et noir les rôles respectifs de l'Église et de l'État (Bélanger, 1987). Fort curieusement, Antoine Prost (1992), parlant des sociologues français qui utilisent la longue durée, arrive aux mêmes conclusions en montrant l'apport de l'approche sociologique pour éclairer des mésinterprétations de l'avènement des lois Ferry en France.

L'amnésie historique caractéristique de la periode post-Révolution tranquille pouvait en partie s'expliquer par l'impatience des réformateurs et des penseurs qui visaient à faire «du passé table rase». Dans tout processus révolutionnaire, même tranquille, le souci de construire du neuf fait temporairement oublier les fondations ou les fondements des approches nouvelles. Inversement, l'intérêt actuel des sociologues pour la longue durée semble s'expliquer de diverses façons. Sommes-nous actuellement à l'ère des paradigmes perdus, comme le pensent Dandurand et Ollivier (1987) ou sombre-t-on simplement dans la nostalgie ou encore, est-on confronté à un phénomène de génération? Certes, une certaine désillusion, voire la grisaille de cette période de crise qui n'en finit pas, favorise un retour sur le passé dans le souci de comprendre l'origine de nos problèmes actuels. Antoine Léon (1980) émet d'ailleurs l'hypothèse que l'émergence d'une réflexion historique résulte quelquefois du sentiment de la nécessité d'agir sur son destin ou encore, de faire le point en situation de crise dans une société faisant face à un blocage social.

Après des appels répétés et réguliers à l'interdisciplinarité, et le fait que de nombreuses recherches empruntent aux deux disciplines, il importe de se questionner sur les rapports mutuels qu'elles entretiennent entre elles et sur ce que l'on peut qualifier d'approche socio-historique. Nos penchants mutuels peuvent-ils faire en sorte que nous nous rejoignons plus que nous le faisons actuellement? Comment en effet dépasser la juxtaposition de deux méthodes, de deux paradigmes?

\section{L'Histoire peut-elle tester l'objectivité de la Sociologie?}

Dans un article fort intéressant, Nadia
Fahmy-Eid $(1991)^{6}$ propose que l'Histoire serve à tester des théories scientifiques ${ }^{13}$. Dans quelle mesure peut-on appliquer cette idée dans le domaine de l'éducation? C'est ce que j'analyserai dans la partie qui suit, en débutant par un questionnement quelque peu lapidaire: L'Histoire de l'Education a-t-elle plus fait avancer notre connaissance de l'appareil scolaire que 40 ans de Sociologie de l'Éducation?

Depuis des décennies, les sociologues de l'éducation tentent de démontrer, ou au contraire de réfuter, selon le cadre d'analyse choisi, la thèse à l'effet que l'éducation contribue à la perpétuation des classes sociales ou de l'inégalité des chances. Analyses régressives, longitudinales ou autres sont mises dans la balance pour appuyer de telles allégations. Pendant ce temps, d'autres études se penchaient en profondeur sur-les idéologies éducatives ou encore sur la place que l'Église accorde aux femmes en éducation, sur l'école en tant que véhicule de l'idéologie de la classe dominante, appareil de régulation, de moralisation de la classe ouvrière, ou au contraire, devant ouvrir l'esprit des classes autrefois défavorisées. Ce n'était pas du temps perdu, loin s'en faut, mais pendant ce temps, notre connaissance de notre passé scolaire restait néanmoins lacunaire. Nous n'y portions pas attention, trop occupés à mesurer nos acquis en matière de démocratisation, ou encore rassurés d'assister à la débâcle de l'Église en éducation. Concurremment, avec la patience de bénédictin qui les caractérise, historiens et historiennes de l'éducation compilaient les effectifs de certaines filières scolaires, par exemple le primaire supérieur, ou encore la présence féminine au cours lettres-science, à l'aide d'archives parfois difficiles à trouver et souvent fastidieuses à consulter. Ces exercices, pour comptables qu'ils puissent apparaître à première vue, étaient à la base d'un nouvel éclairage sur l'école.

Loin de moi l'idée d'encenser les derniers et de rejeter le travail des premiers. Ce serait vite oublier mes origines et mon orientation première en Sociologie de l'Éducation. Mais lors de la lecture des travaux du collectif Clio sur l'histoire des fernmes (1982) ou encore devant l'importance et la qualité

\footnotetext{
" Cet article a d'ailleurs obtenu lê prix Hilda Neatby du comité Canadien de 1 'Histoire des Femmes (CCHF) en 1992.

${ }^{13}$ Cada uma das participantes quis responder a um questionário confidencial
}

Paidéia, FFCLRP-USP, Rib. Preto, jan/julho/2000. 
des séances relatives à l'Histoire de l'Éducation au congrès de la Société Historique du Canada en 1985, il m'apparaissait que plusieurs travaux d'historiens et d'historiennes éclairaient d'un jour particulier des facettes de l'évolution de l'appareil scolaire, au moins autant que plusieurs années en Sociologie de l'Éducation. Cet enthousiasme provenait-il de la nouveauté d'un champ jusqu'alors exploré à petits pas? Non, mais simplement du fait que l'analyse concrète de quelques filières éducatives permettait de mieux comprendre les mécanismes de socialisation en vigueur dans certaines des institutions scolaires qui ont précédé l'organisation des polyvalentes et des CÉGEPS, créés par la Révolution tranquille.

Un voile commençait à s'ouvrir, avec pour conséquence une meilleure compréhension des flux de fréquentation scolaire. Soudain, on apprenait enfin ce que représentaient les écoles «sous-contrôle et indépendantes» si souvent mentionnées dans les Rapports du Surintendant de l'Instruction Publique. Grâce aux travaux d'historiennes de l'éducation, il apparaissait au grand jour que l'enseignement privé des religieuses avait entre autres eu pour effet de permettre de financer les écoles publiques où oeuvraient ces mêmes communautés enseignantes (Malouin, 1983). L'interprétation des rapports ÉgliseÉtat, autrefois antagoniques, prenaient ainsi une tout autre configuration. On en venait à réinterpréter les rapports entre institutions privées et publiques, les premières ne pouvant être totalement amalgamées à des écoles de riches à l'usage exclusif des bien-nantis, étant donné qu'une partie des recettes de ces dernières servait à financer les filières accessibles aux plus pauvres, filières le plus souvent organisées par la même communauté enseignante. De même, les rapports entre hiérarchie religieuse et communautés de bases prennent une tout autre allure lorsqu'on connaît les luttes épiques des communautés religieuses de femmes pour l'obtention des collèges classiques féminins devant l'opposition des Évêques? ${ }^{7}$.

Ces quelques exemples me servent d'illustration pour pousser plus loin mon propos sur

\footnotetext{
'L'histoire sert aussi à réinterpréter la Révolution tranquille, comme d'ailleurs des périodes plus lointaines. Ainsi, dans um article percutant publié em 1987, Charlant replace l'avènement des premières lois scolaires de la dernière tranche du siècle dernier, a dû composer avec um courant liberal minoritaire mais existant, contrairement à d'autres interprétations de cette période qui laissent entendre que les ultamontains dominaient majoritairement
}

Paidéia, FFCLRP-USP, Rib. Preto, jan/julho/2000. l'apport de l'Histoire de l'Éducation à notre connaissance de notre appareil scolaire. En effet, s'il est un secteur de l'Histoire qui a énormément fait avancer notre compréhension de notre système scolaire, c'est le secteur de l'éducation des filles grâce aux travaux dirigés principalement par Micheline Dumont (Dumont \& coll., 1986) et Nadia FahmyEid (Fahmy-Eid \& coll., 1983). Leurs ouvrages collectifs, ainsi que l'impressionnante production de leurs équipes de travail, apportent une contribution inestimable à l'Histoire de l'Éducation Québécoise (Gaffield, 1985). Ceci fut souligné à maintes reprises particulièrement lors de la communication de Chad Gaffield à la Société Historique du Canada en 1985 et lors d'un bilan des recherches portant sur l'éducation des filles, effectué par Renée Cloutier; Roberta Mura \& France Parent (1987). Ces travaux de la part d'historiennes enrichissent notre compréhension en avançant dans le domaine de l'interprétation. Le collectif Clio, qui pourtant ne porte pas exclusivement sur l'éducation des filles, va encore plus loin en proposant des éléments visant à réinterpréter la Révolution tranquille. À titre d'exemple, dans la première édition de cet ouvrage, on se demandait si la Révolution tranquille ne s'était pas faite sur le dos des femmes. Ce à quoi des travaux récents permettent de répondre par l'affirmative. Nous pensons ici entre autres aux travaux de Claudine Baudoux $(1989,1991)$ sur la féminisation de la gestion en éducation ou même sur les Écoles Normales (Hamel, 1991; Baudoux, 1989, 1991).

Les travaux sur l'éducation des filles, ou encore ceux relatifs aux Frères enseignants, représentent des exemples parmi d'autres de travaux dont l'objet est l'Histoire de l'Éducation, mais où le souci d'interprétation et surtout la prise en compte du contexte social et politique ainsi que des facteurs sociaux pouvant induire des comportements, ou encore influencer les contenus enseignés, ont réussi à faire le pont entre Histoire et Sociologie. Dans le cas du secteur éducatif, l'idée lancée par Nadia Fahmy-Eid (1991) à l'effet que l'Histoire puisse aider à tester l'objectivité de certaines théories m'apparaît donc fort pertinente, particulièrement en nous permettant d'affiner notre façon d'entrevoir le rôle de l'école dans la société et surtout de réinterpréter la Révolution tranquille. Mais nous pouvons pousser plus loin notre questionnement et nous demander si 
le retour au passé est essentiel à la compréhension des problèmes actuels de notre système scolaire. Le regard sociologique enrichi par la longue durée apporte-t-il un éclairage espécifique tel que nous puissions dire qu'une approche sociologique éclairée par l'Histoire peut aider à agir sur le présent?"

\section{Un parti pris: le passé éclaire l'avenir ou le passé et son avenir}

"Toutes les questions posées au passésont filles de leurs temps. »

Au fil du temps qui passe, les systèmes éducatifs changent, évoluent, se modifient. Certaines périodes paraissent à première vue propices à des changements en douceur alors que d'autres représentent des cassures par rapport à la période antérieure. Dans la période actuelle de transformations sociales, économiques et politiques intenses que nous connaissons, et qui traversent aussi et nécessairement l'appareil scolaire, il importe de comprendre les changements en éducation. Or pour le faire, nous croyons fermement qu'il est nécessaire de recourir au passé. Cette démarche présente plusieurs avantages dont celui de relativiser notre regard sur l'école. Comme le dit Antoine Léon, éviter les engouements passagers et comprendre les racines des problèmes actuels. En outre, l'analyse sociohistorique permet de démystifier les jugements portés sur le système éducatif.

En résumé, l'approche historique des faits éducatifs représente un outil indispensable d'analyse des situations présentes grâce à un souci de relativiser - sans verser dans le pyrrhonisme ou le relativisme incohérent - les problèmes actuels et à la préoccupation d'éclairer les comportements et le fonctionnement des institutions en leur restituant leur dimension temporelle (Léon, 1980, p. 15).

Mais malgré tout, peut-on faire l'économie de ce retour historique? Braudel insiste longuement sur le rapport au temps qui semble distinguer historiens et sociologues. Les historiens n'y

\footnotetext{
${ }^{8}$ L'hisotien qui pousse très loin l'interprétation aux moyens de la conceptualisation qu'apportent les sociologues, ou encore lê sociologue quei travaille avec la longue durée donnent-ils les mêmes résultats?
}

échappent pas alors que les sociologues tentent de s'y soustraire soit en travaillant sur le temps extrêmement court des enquêtes ou, au contraire, en cherchant à établir des structures intemporelles. Pourtant, comme le montre Braudel, inévitablement, pour qualifier un modèle, une structure, les sociologues doivent peu à peu intégrer le temps et c'est le temps long qui semble le plus propice à dégager des tendances. La notion de la longue durée, point d'ancrage commun aux historiens et à certains sociologues, permet de relativiser notre regard sur le présent tout en se préservant des engouements passagers et -non fondés. C'est aussi la prise en compte du temps long qui permet de cerner les discontinuités et aussi les continuités qui traversent une même question scolaire. La longue durée ne représente toutefois pas le seul élément d'affinités entre historiens et sociologues. La dialectique de la durée permettrait-elle, comme le pense Braudel, d'être sensible aux intempéries de l'Histoire?

En retournant au passé, comme le montre très bien Antoine Léon, on peut avoir l'illusion que tout a été dit. Au contraire, on peut avoir l'illusion de la nouveauté d'un phénomène, la notion de l'inédit, alors que cette impression peut être seulement. due à notre méconnaissance d'une réforme aujourd'hui oubliée. C'est là que l'Histoire de l'Éducation démontre si pertinence et son apport indéniable. L'Histoire de l'Éducation pourra ainsi donner un peu plus de rigueur à nos interprétations et éviter certains lieux communs qui tiennent fréquemment lieu d'analyse en éducation. Le détour historique peut ainsi nous faire redécouvrir une réforme en germe, qui n'a pu se concrétiser parce que non reprise par les réformateurs alors au pouvoir, mais qui pourra à une autre époque devenir le concept intégrateur, point de départ ou de rupture d'une autre période éducative.

La façon de faire l'Histoire varie en outre selon les contextes donnés, les buts poursuivis et le groupe qui fait ou «commande» l'Histoire. Celle-ci peut fort bien servir à justifier le passé ou au contraire le dépasser. Dans une période qualifiée de révolutionnaire ou de rupture, on peut citer le passé pour rejeter un mode d'organisation sociétal. C'est ce que le Québec a plus ou moins vécu suite à la Révolution Tranquille. Par contre, dans la période actuelle, grâce entre autres aux travaux de nature sociohistorique, la façon d'interpréter le rôle de

Paidéia, FFCLRP.USP, Rib. Preto, jan/julho/2000. 
l'Église en éducation est en train de changer fondamentalement. Il semble que l'apport du clergé en éducation ne soit plus vu seulement comme négatif et réactionnaire, comme au temps de la réforme, mais au contraire comme ayant apporté aussi certaines contributions inestimables. Quelle serait la place qu' aurait occupée l'éducation des filles s'il n'y avait pas eu l'apport des religieuses enseignantes? Mais qu'on le veuille ou non, nous sommes toujours tributaires, d'une façon ou d'une autre, de notre histoire passée. C'est donc dire qu'à la question: le retour au passé est-il incontournable, nous répondons comme Antoine Léon (1980, p. 9): «La fréquentation du passé aide à dégager l'originalité du présent.»

\section{Pour de nouveaux rapports entre Histoire et Sociologie}

«L'historien comme le sociologue ont à s'interroger sur la façon dont ils articulent, dans leurs recherches respectives, deux formes de raisonnement apparemment contradictoires,» (Prost, 1992, $p, 03)$

Ainsi depuis une quinzaine d'années, l'Histoire de l'Éducation tant au Québec qu'au Canada est un domaine où l'avancement des recherches fait des bonds prodigieux. Diverses facettes de notre histoire scolaire, autrefois à peine explorées, commencent à être étudiées en profondeur. Du côté de la Sociologie de l'Éducation, qui a vécu ses heures de gloire à partir des années soixante-dix, on est actuellement à redéfinir la façon d'appréhender l'étude de l'école et des rapports avec la société. Les thèmes abordés, le type de recherche effectuée sont en train d'évoluer vers de nouvelles approches à la fois théoriques et méthodologiques. Jadis partagés en deux champs disciplinaires, ces deux secteurs d'activités autrefois relativement indépendants et étanches, sont en train de s'interpeller mutuellement. Des sociologues de l'éducation se penchent et investissent le champ de l'Histoire et inversement, des historiens s'intéressent de plus en plus à la Sociologie ou du moins aux Sciences Sociales. À cet égard, l'Histoire et la Sociologie de l'Éducation sont actuellement dans une période charnière où de nouvelles perspectives sont en train d'émerger. La période actuelle est sans contredit un moment

Paidéia, FFCLRP-USP, Rib. Preto, jan/julho/2000. important de définition de nouveaux champs de recherches, de nouvelles relations entre Histoire et Sociologie. Nos penchants réciproques ne pourraientils pas faire en sorte que nous nous rejoignions plus que nous ne le faisons actuellement?

Nous sommes dans une période intense de crise des idéologies et des modèles où l'on est à la recherche de nouveaux paradigmes porteurs d'avenir. Je la qualifierais en outre de période de maturité dans le domaine de la recherche en Éducation où, grâce à l'apport- mutuel de l'Histoire et de la Sociologie, nous pouvons dépasser les deux «tendances manichéennes» identifiées par Micheline Dumont. Cette maturité, en permettant de réinterpréter sans remords la Révolution Tranquille, nous permettra d'avancer pour ainsi mieux comprendre les enjeux éducatifs que les prochaines décennies nous réservent. À l'aube de l'an 2000 , il nous faudra beaucoup d'imagination sociologique et de solides bases historiques pour négocier les prochains virages éducatifs qui nous attendent au détour du chemin.

\section{References}

Audet, L.-P. (1964). Histoire du Conseil de l'instruction publique 1856-1964. Montréal : Léméac.

Audet, L.-P. (1951). Le système scolaire de la province de Québec. Québec: Les éditions de l'érable, 6 tomes.

Audet, L.-P. (1971). Histoire de l'enseignement au Québec, 1840-1971. Montréal : Holt, Rinehart et Winston Ltée, Tome 1 et 2.

Baudoux, C. (1991). Effets de la laïcisation et de la mixité sur le nombre de femmes responsables d'établissements scolaires du Québec de 1958 à 1985. Revue des sciences de l'éducation, XVII (1), 1-24.

Baudoux, C. et al. (1989). Féminisation et masculinisation de la gestion. Sainte-Foy, Groupe de recherche multidisciplinaire féministe, Université Laval: Laval

Bélanger, P. (1988). Educational Reform in Quebec. Dans T., Lorne \& J. Curtis (Org.), Readings in Sociology, (pp.554-564). An Introduction. Toronto: McGraw-Hill/Ryerson.

Bélanger, P.W. (1987). Rétrospective de la recherche en éducation en milieu francophone canadien. 
Recherche et progrès en éducation. Bilan et prospective. Actes du ler congrès des sciences de l'éducation de langue française du Canada. Québec: Faculté des Sciences de l'Éducation.

Braudel, F. (1969). Ecrits sur l'histoire. Paris : Flammarion.

Charland, J.P. (1987). Le réseau d'enseignement public Bas-Canadien, 1841-1867: une institution de l'État libéral». R.H.A.F. ,40 (4), 505-535.

Charland, J.-P. (1982). Histoire de l'enseignement professionnel et technique. Québec: Institut Québécois de Recherche sur la Culture.

Cloutier, R. ; Roberta, M. \& France, P. (1987). Tendances et perspectives de la recherche sur les femmes et l'éducation au Canada 1975-1986. Recherche et progrès en éducation. Bilan et prospective. Actes du premier Congrès des sciences de l'éducation de langue française $d u$ Canada. Québec: Faculté des Sciences de l'Éducation.

Collectif, C. (1992). L'histoire des femmes au Québec depuis quatre siècles. Édition entièrement revue et mise à jour, Montréal: Le Jour Éditeur.

Dandurand, P. (1984). La recherche en sociologie de l'éducation au Québec. Prospectives. Février 1984: p. 69-75.

Dandurand, P. (1992). L'éducation et la sociologie de l'éducation au Québec de 1950 à 1990. dans Plaisance, Eric, dir. Programme et renouvellement en sociologie de l'éducation. (95102). Paris: INRP/L'Harmattan.

Dandurand, P. \& Emile, O. (1987). Les paradigmes perdus. Essai sur la sociologie de l'éducation et son objet. Sociologies et sociétés, Octobre, vol XIX (2), 87-103.

Dandurand, P. \& Roland, O. (1990). Les grandes orientations de la recherche en sociologie de l'éducation au Québec: un bilan bibliographique. Québec: Les Cahiers du LABRAPS.

Depaepe, M. (1983). L'apport de l'histoire de l'éducation à la définition des politiques éducatives. Quelques réflexions méthodologiques. Dans Willem Frijhoff, dir. L'affre d'école. Paris: Publication de la Sorbonne, INRP.

Dumont, M. (1990). L'instruction des filles au
Québec (1639-1960). Ottawa: La Société Historique du Canada, Brochure Historique.

Dumont, M. \& Fahmy-Eid, N. (1986). Les couventines, l'éducation des filles au Québec dans les congrégations religieuses enseignantes, 1840-1960. Montréal : Boréal.

Dumont, M. \& Fahmy-Eid, N. (1991). L'histoire de l'éducation des filles, La pointe de l'Iceberg». Revue d'histoire de l'éducation/ Historical Studies in Education, 3(2), 211-237.

Eid, N.F. (1978). Le clergé et le pouvoir politique au Québec: une analyse de l'idéologie ultramontaine au milieu du XIXe siècle. Montréal:Hurtubise HMH, Collection Histoire.

Fahmy-Eid, N. (1991). Histoire, objectivité et scientificité. Jalons pour une reprise de débat épistémologique. Histoire sociale/Social History. Vol. XXIV (47), 9-34.

Fahmy-Eid, N. \& Dumont, M. (1983). Maîtresses de maison, maîtresses d'école. Femmes, famille et éducation dans l'histoire du Québec. Montréal: Boréal Express.

Fahmy-Eid, N., Dumont, M. \& Heap, R. (1988). Rapport spécial sur le Québec. 1. Bilan des publications et recherches en histoire de l'éducation au Québec au cours des quatre dernières années. 2. l'Histoire de l'éducation au Québec: Perspectives critiques». Bulletin d'ACHE, 2, 59-71.

Galarneau, C. (1978). Les collèges classiques au Canada-français. Montréal, Fides.

Graff, H.J. (1991). Towards 2000: Poverty and Progress in the History of Education. Revue d'histoire de l'éducation/Historical Studies in Education, 3(2), 191-211.

Hamel, T. (1991). Le déracinement des écoles normales, Québec: Institut Québécois de Recherche sur la Culture.

Hamel, T. (1995). Un siècle de formation des maîtres au Québec. 1836-1939. Montréal: Hurtubise HMH.

Heap, R. (1986). L'Église, l'État et l'enseignement primaire catholique au Québec, 1897-1920. Thèse de doctorat, Université de Montréal.

Henchy, N. \& Burgess, D. (1987). Between Past and Future. Quebec Education in transition. Calgary:

Paidéia, FFCLRP-USP, Rib. Preto, jan/julho/2000. 
Detselig Enterprises Limited.

Isambert-Jamati, V. (1970). Crises de la société. Crises de l'enseignement, Paris: PUF.

Katz, M.B. (1971). Class, Bureaucracy and Schools. The Illusion of Educational change in America. New York: Praeger Publishers.

Lefebvre, B. (1980). L'école sous la mître. Montréal: Les Éditions Paulines.

Léon, A. (1980). Introduction à l'histoire des faits éducatifs. Paris: PUF.

Magnuson, R. (1969). The decline at Roman Catholic education in Quebec. Interpretation and explorations. Culture, XXX (3),192-198.

Mair, N.H. (1981). Protestant Education in Québec. Notes on the History of Education in the Protestant public schools of Québec. Québec: Conseil Supérieur de l'Éducation.

Malouin, M.-P. (1989). Que sont devenues les soeurs de nos écoles? Recherches sur les orientations actuelles des religieuses enseignantes au Québec. Québec: AREQ.

Malouin, M.-P. \& Dumont, M. (1986). L'évolution des programmes d'études (1850-1960). Dans $\mathrm{M}$. Dumont \& N. Fahmy-Eid.(Orgs.), Les couventines, l'éducation des filles au Québec dans les congrégations religieuses enseignantes, 1840-1960, (83-112). Montréal: Boréal.

Parent, F.; Cloutier, R. \& Mura, R. (1988). Recherches sur les femmes et éducation formelle au Canada et au Québec publiées dans la période 1975-1986. Recherches féministes, 1(1), 129-149.

Petitat, A. (1982). École et sociétés: le paradigme de la reproduction et ses limites. Revue européenne des sciences sociales, $X X(63), 5-27$.

Petitat, A. (1982). Production de l'école-Production de la société. Analyse socio-historique de quelques moments décisifs de l'évolution scolaire en occident. Genève: Librairie Droz.

Phillips, C.E. (1957). The Development of Education in Canada. Toronto: W.J. Gage and Co. Ltd..

Piquette, R. (1973). Les programmes de formation des maîtres dans les écoles normales françaises du Québec (1857-1970). Thèse de doctorat, Université de Montréal.

Plaisance, E. (1992a). Permanence et renouvellement

Paidéia, FFCLRP-USP, Rib. Preto, jan/julho/2000. en sociologie de l'éducation. Perspectives de recherches. 1950-1990. Paris: L'Harmattan'.

Plaisance, E. (1992b). Sociologie et sciences de l'éducation. Continuités historiques et spécificités de l'apport de Viviane IsambertJamati. dans Plaisance, Eric, dir. Permanence et renouvellement en sociologie de l'éducation, Paris: L'Harmattan.

Prost, A. (1992). Lecture historique et lecture sociologique des politiques d'éducation. Dans E. Plaisance (org.), Programme et renouvellement en sociologie de l'éducation, (pp. 203-212). Paris: L'Harmattan.

Recherche et progrès en éducation. Bilan et prospective. Actes du ler congrès des sciences de l'éducation de langue française du Canada. (1987).Québec, Faculté des Sciences de l'Éducation, 416 pages.

Simon, B. (1984). Can Education Change Society. In : J.D. Wilson (Ed.). An Imperfect Past. Education in Canadian history, (pp.30-38). Columbia: Center for the Study of Curriculum and Instruction ed.

Thivierge, N. (1982). Écoles ménagères et instituts familiaux: un modèle féminin traditionnel. Québec: Institut Québécois de Recherche sur la Culture.

Thivierge, M. (1981). Les institutrices laïques à l'école primaire catholique au Québec de 1900 à 1964, Thèse de doctorat, Université Laval.

Tremblay, A. (1989). Le ministère de l'Education et le Conseil supérieur, antécédents et création, 1867-1964. Sainte-Foy: Presses de l'Université Laval.

Trottier, C. (1987). La nouvelle sociologie de l'éducation. Québec: Les Cahiers du Labraps, Série Etudes et documents, éd.

Turcotte, P.-A. (1981).L'éclatement d'un monde: les Clercs de Saint-Viateur et la révolution tranquille. Montréal: Éditions Bellarmin.

Turcotte, P.-A. (1985). Les chemins de la différence. Pluralisme et aggiornamento dans l'aprèsconcile. Montréal: Éditions Bellarmin.

Turcotte, P.-A. (1988). L'enseignement secondaire public des Frères éducateurs (1920-1970). Utopie et modernité. Montréal: Éditions Bellarmin. 
Turcotte, P.-A. (1993). «Sociologie et histoire. Les jeux et enjeux d'une pratique». Eglise et théologie.

Wexler, P. (1987). Social Analysis of Education after the New Sociology. New-York: Routledge.

Wilson, J.D. (1984a). Somes Observations on Recent Trends in Canadian Educational History». J.D. Wilson. An Imperfect Past. Education and society in Canadian History. Vancouver: Canadian History of Education Association/Center for the Study of Curriculum and Instruction.

Wilson, J.D. (1984b). An imperfect Past. Education and Society in Canadian History. Vancouver: Association Canadienne d'Histoire de l'Éducation.

Wilson, J.D. (1984c). Introduction». J.D. Wilson. An Imperfect Past. Education in Canadian history. Columbia: Center for the Study of Curriculum and Instruction.

Nota: Artigo originalmente publicado na revista Hisotire et Sociologie e reproduzido com o aval da revista 